\title{
The relationship between aspirin use and mortality in colorectal cancer
}

\author{
Heidar J. Albandar ${ }^{1}$, Ronald Markert ${ }^{1}$, Sangeeta Agrawal ${ }^{1,2}$ \\ ${ }^{1}$ Department of Internal Medicine, Wright State University Boonshoft School of Medicine, Dayton, OH, USA; ${ }^{2}$ Department of Internal Medicine, \\ Division of Gastroenterology, Dayton VA Medical Center, Dayton, OH, USA \\ Contributions: (I) Concept and design: HJ Albandar; (II) Administrative support: HJ Albandar, S Agrawal; (III) Provision of study materials or patients: \\ HJ Albandar; (IV) Collection and assembly of data: HJ Albandar, R Markert; (V) Data analysis and interpretation: HJ Albandar, R Markert; (VI) \\ Manuscript writing: All authors; (VII) Final approval of manuscript: All authors. \\ Correspondence to: Heidar J. Albandar, MD. 1 Medical Center Dr, Morgantown, WV 26506, USA. Email: hman11@gmail.com.
}

\begin{abstract}
Background: Aspirin use lengthens survival in patients with colorectal cancer (CRC). We aimed to add to the evidence examining this relationship and to compare the survival benefit between aspirin started before CRC diagnosis with those started after CRC diagnosis.

Methods: The study involved 173 consecutive patients who had a histopathologic diagnosis of colorectal carcinoma between 1996 and 2014. The electronic medical record was used to collect data on demographic characteristics (age, sex, and race), family history of cancer, use or non-use of aspirin before and after cancer diagnosis, cancer stage at diagnosis, and days from cancer diagnosis to death. Patients were divided into aspirin using and non-aspirin using groups.

Results: Aspirin users (ASAU, $\mathrm{n}=90$ ) were older than non-aspirin users (NASAU, $\mathrm{n}=83 ; 70.8 \pm 9.1 \mathrm{vs}$. $66.2 \pm 10.2$ years; $\mathrm{P}=0.004$ ). The two groups were similar in sex, race, and family history of cancer, but the non-users of aspirin were more likely to have stage III or stage IV CRC (NASAU $=57.5 \%$; ASAU $=38.6 \%$; $\mathrm{P}=0.014$ ). Aspirin users survived more than twice the number of days than non-users (ASAU median $=941$ days; NASAU median =384 days; $\mathrm{P}=0.003$ ). Patients who used aspirin only before their CRC diagnosis had a short survival period from diagnosis to death (median $=149$ ).

Conclusions: Our findings support the relationship of aspirin use and duration of use with enhanced survival in patients with CRC. Physicians may want to recommend that patients at increased risk for CRC and those already diagnosed, but not yet on aspirin, start an aspirin regimen.
\end{abstract}

Keywords: Malignancy; risk; preventive

Submitted Jun 30, 2018. Accepted for publication Aug 07, 2018.

doi: 10.21037/jgo.2018.08.13

View this article at: http://dx.doi.org/10.21037/jgo.2018.08.13

\section{Introduction}

Colorectal cancer (CRC) is the second most common cause of cancer-related death worldwide (1). While other cancers are expected to decrease in incidence over the next 10 years, CRC is projected to be one of the top four cancer killers until at least 2030 (2). The death rate trend for CRC in the 30-year period between 1975 and 2004 declined for both sexes in most races in the U.S. (3). The incidence rate of CRC has gradually declined over the past 20 years even as the prevalence has increased; largely due to an aging population and greater adherence to screening practices (3). Major risk factors for CRC include male sex, black race, and older age (4). Other risk factors include a previous history of colonic polyps, inflammatory bowel disease, and lifestyle factors such as alcohol/tobacco consumption (5). Factors that reduce the risk of CRC include colonoscopy screenings, high-fiber diet, vegetarian diets, and aspirin use $(6,7)$. Of these factors, only aspirin use may lengthen the survival rate in patients with already existing CRC (8). 
Table 1 Characteristics of 173 patients with colorectal cancer at Dayton Veterans Affairs Medical Center

\begin{tabular}{|c|c|c|c|}
\hline Characteristic & ASAU $(n=90)$ & NASAU $(n=83)$ & $P$ value \\
\hline Sex (male), n (\%) & $84(93.3)$ & $79(95.2)$ & 0.85 \\
\hline Race $^{*}$ (Caucasian), n (\%) & $78(86.7)$ & $70(87.5)$ & 0.87 \\
\hline Family history of cancer ${ }^{\dagger}, \mathrm{n}(\%)$ & 27(33.3) & $30(42.9)$ & 0.23 \\
\hline Stage I or II & $54(61.4)$ & $34(42.5)$ & \\
\hline Stage III or IV & $34(38.6)$ & $46(57.5)$ & \\
\hline Days from CRC diagnosis to death, median & 941 & 384 & 0.003 \\
\hline
\end{tabular}

*, Caucasian compared to African-American. Three patients with a race of other excluded. ${ }^{\dagger}$, data unavailable for 22 patients. ${ }^{\ddagger}$, data unavailable for 5 patients. ASAU, aspirin users before and/or after diagnosis of colorectal cancer; NASAU, non-users of aspirin before or after diagnosis of colorectal cancer.

Most CRCs arise in an adenoma, and a $19 \%$ relative risk reduction of recurrent adenomas and a $40 \%$ relative risk reduction of advanced adenomas among aspirin (ASA) users have been reported (9). A review found that aspirin reduced the risk of developing CRC (10), establishing the chemopreventive role of ASA for CRC (11). The effect of ASA on the prognosis of CRC has also been studied. Use of ASA after diagnosis of stage III colon cancer has demonstrated mortality benefit (12). Among patients diagnosed with non-metastatic CRC participating in the Nurses' Health Study and Health Professionals Follow-up Study, ASA use after diagnosis of CRC lowered the risk of CRC-specific and all-cause mortality (13).

The aim of this study was to investigate the relationship between aspirin use and CRC survival. Our goal was to determine if there was a mortality benefit in veterans with CRC who (I) initiated aspirin after cancer diagnosis or (II) were already on aspirin before their cancer diagnosis.

\section{Methods}

This retrospective study was conducted at the Dayton (Ohio) Veterans Affairs Medical Center (DVAMC). The DVAMC provides multidisciplinary care for more than 40,000 patients who served in the United States Armed Forces. At the DVAMC cancer screening, diagnosis, and management are collectively carried out by multiple departments, including gastroenterology, interventional radiology, radiation oncology, medical oncology, surgical oncology, and palliative/hospice care. The study was approved by both the Wright State University Institutional Review Board and the DVAMC Research and Development Committee.

The study involved 173 consecutive patients who had a histopathologic diagnosis of colorectal carcinoma between 1996 and 2014. The electronic medical record was used to collect data on demographic characteristics (age, sex, and race), family history of cancer, use or non-use of aspirin before and after cancer diagnosis, cancer stage at diagnosis, and days from cancer diagnosis to death. All authors had access to the study data and had reviewed and approved the final manuscript. Patients were divided into aspirin using and non-aspirin using groups That included non-users of aspirin, aspirin user before and after CRC diagnosis, aspirin users only before CRC diagnosis, and aspirin users only after CRC diagnosis. Staging of the patient's CRC followed the classification system of the American Joint Committee on Cancer (AJCC). Patients with incomplete documentation on aspirin use were excluded.

The Mann-Whitney (M-W) Test, Kruskal-Wallis Test (K-W), Chi-square test, Kaplan-Meier survival analysis, and multiple linear regressions were used for analyses (IBM SPSS Statistics 23.0). The K-W test was utilized in analysis of the four aspirin groups whereas the $M-W$ test was utilized for comparing ASAU and NASAU. These tests were used because the outcome variable, days from CRC diagnosis to death, was not normally distributed.

\section{Results}

A total of 173 patients with a histopathologic diagnosis of CRC were grouped based on aspirin use $(n=90)$ or no use of aspirin before or after CRC $(\mathrm{n}=83)$. Table 1 shows 
that aspirin users (ASAU) were older than non-users of aspirin (NASAU) $(70.8 \pm 9.1$ vs. $66.2 \pm 10.2$ years; $\mathrm{P}=0.004)$. The two groups were similar in sex (ASAU $=93.3 \%$ male; NASAU $=95.2 \%$ male; $\mathrm{P}=0.85$ ) and race (ASAU $=86.7 \%$ Caucasian; NASAU =87.5\% Caucasian; $\mathrm{P}=0.87)$. The two groups did not differ on family history of cancer (ASAU $=33.3 \%$; NASAU $=42.9 \% ; \mathrm{P}=0.23$ ), but the nonusers of aspirin were more likely to have stage III or stage IV CRC (NASAU =57.5\%; ASAU =38.6\%; $\mathrm{P}=0.014$ ).

Aspirin users survived more than twice the number of

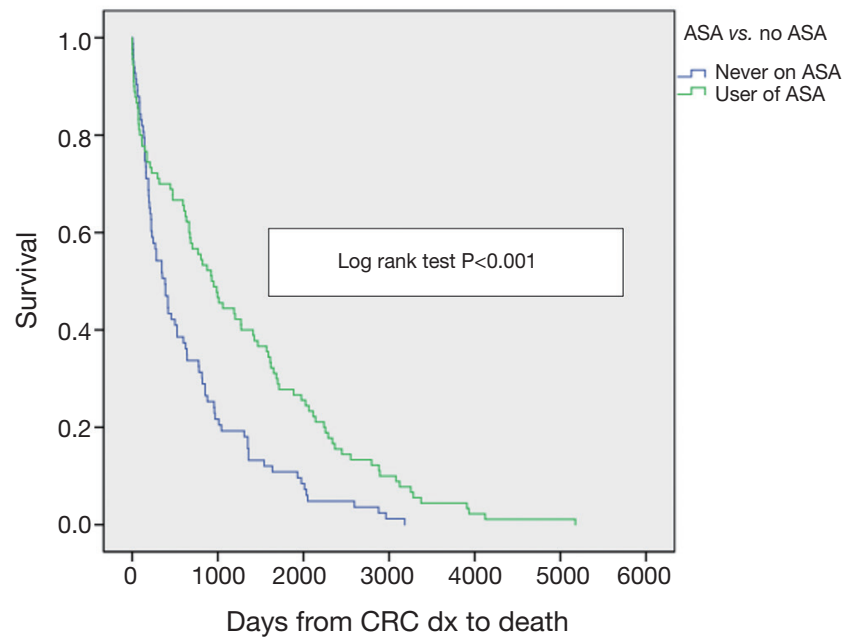

Figure 1 Kaplan-Meier survival for aspirin users and non-users of aspirin. days than non-users (ASAU median $=941$ days; NASAU median $=384$ days; $\mathrm{P}=0.003$ ). After adjusting for the two characteristics significantly different between the two groups on a univariate basis (age and stage of cancer), the difference between ASAU and NASAU changed little. The level of significance fell from 0.003 to 0.001 and the mean survival for the 173 patients increased from 972 to 989 days. Kaplan-Meier analysis shows the better survival experience for the aspirin users compared to the non-users $(\mathrm{P}<0.001)$ (Figure 1).

Table 2 reports the characteristics by four subgroups, i.e., NASAU, aspirin user before and after diagnosis, aspirin user only before diagnosis, and aspirin user only after diagnosis. Of note is the short survival for those who used aspirin only before their CRC diagnosis (median $=149$ ).

\section{Discussion}

This study shows that the ASAU group lived more than twice as long as the NASAU following diagnosis of CRC (median survival: 941 vs. 384 days). The survival gain for ASAU may have been aided by their age advantage (i.e., 4.6 years younger on average) and having less advanced CRC. However, after adjusting for age and stage of cancer, the difference between the two groups changed little, and remained statistically significantly different.

Our finding of a longer survival rate for patients who used aspirin before and after their CRC was diagnosed is

Table 2 Characteristics of four groups with colorectal cancer at Dayton Veterans Affairs Medical Center

\begin{tabular}{|c|c|c|c|c|c|}
\hline Characteristic & NASAU $(n=83)$ & $\begin{array}{l}\text { ASA before/after } \\
\text { CRC }(n=41)\end{array}$ & $\begin{array}{l}\text { ASA before CRC } \\
(n=27)\end{array}$ & $\begin{array}{l}\text { ASA after CRC } \\
\quad(n=22)\end{array}$ & $P$ value \\
\hline Age, years, mean $\pm S D$ & $66.2 \pm 10.2$ & $70.4 \pm 8.2$ & $72.0 \pm 10.2$ & $70.1 \pm 9.4$ & $0.029^{\mathrm{a}}$ \\
\hline Race $^{\star}$ (Caucasian), n (\%) & $70(87.5)$ & $33(80.5)$ & $24(88.9)$ & $21(95.5)$ & 0.35 \\
\hline Family history of cancer ${ }^{\dagger}, \mathrm{n}(\%)$ & $30(42.9)$ & $7(20.6)$ & $12(46.2)$ & $8(38.1)$ & 0.12 \\
\hline Stage I or II & $34(42.5)$ & $27(65.9)$ & $14(56.0)$ & $13(59.1)$ & \\
\hline Stage III or IV & $46(57.5)$ & $14(34.1)$ & $11(44.0)$ & $9(40.9)$ & \\
\hline Days from CRC diagnosis to death, median & 384 & 987 & 149 & 1197 & $<0.001^{\mathrm{b}}$ \\
\hline
\end{tabular}

${ }^{*}$, Caucasian compared to African-American. Three patients with a race of other excluded. ${ }^{\dagger}$, data unavailable for 22 patients. ${ }^{\ddagger}$, data unavailable for 5 patients. ${ }^{a}$, significant pairwise comparisons: NASAU younger than ASA before/after CRC and ASA before CRC; ${ }^{b}$, significant pairwise comparisons: ASA before/after CRC and ASA After CRC had higher median days than NASAU and ASA Before CRC. ASAU, aspirin users before and/or after diagnosis of colorectal cancer; NASAU, non-users of aspirin before or after diagnosis of colorectal cancer. 
consistent with the literature reporting that extended use of ASA inhibits metastasis of already existent malignancy (14). Reimers et al. have also reported improved survival after starting ASA in patients with human leukocyte antigen (HLA) class 1 antigen expressing CRC tumors (15). The Thrombosis Prevention Trial, using controlled-release formulations of ASA $(75 \mathrm{mg})$, found the greatest reduction in incidence of CRC and mortality after 5 years of daily ASA (8).

Recent studies have focused on the short- and long-term effects of aspirin on the incidence of cancer and resulting mortality $(8,14,16)$. Rothwell et al. determined that the longer the duration of ASA use, the greater the benefit seen with reduction in CRC incidence and mortality (14). In addition to long-term (5 years and greater) benefits, ASA use was found to have short-term mortality benefit within as little as 3 years of follow-up. This finding is corroborated in our study as patients that were started on ASA after CRC diagnosis demonstrated mortality benefit within a median of 3.28 years ( 1,197 days).

Previous studies specifically addressing the effect of ASA use on mortality after diagnosis of CRC have had mixed results. Two observational studies have shown a 30-40\% reductions in CRC-specific mortality when ASA was started after diagnosis of CRC $(13,17)$. But a large population based cohort study showed no improvement in survival time when low-dose aspirin was started after diagnosis of CRC (18).

There are some limitations to our study. First, the study was conducted at a single institution with a group of veterans, nearly all of whom were male. Thus, generalizability to the general population may be restrictive. Second, in retrospective chart reviews data omissions are more likely, and the accuracy of data collection more challenging. Third, the sample size was relatively modest $(n=173)$. However, the nearly equal distribution of the sample between aspirin users (52\%) and non-users (48\%) benefited the statistical analysis. Furthermore, the differences between groups (whether two groups or four groups) were large. Thus, low power due to a modest sample size did not occur. Finally, conclusions from observational studies, such as ours, are susceptible to confounding; however, when we controlled for the two factors on which aspirin users and non-users differed (age and stage of cancer), these adjustments had minimal effect on the findings.

During the past decade the guidelines on primary prevention of CRC have changed. More recently, the U.S. Preventive Services Task Force released recommendations endorsing low-dose aspirin use in adults with cardiovascular disease (CVD) to address primary prevention of CVD and the increased risk for CRC (19). According to these guidelines, aspirin use is most beneficial for the age group 50 to 59 years, whereas there is insufficient evidence for older age groups. In the present study the average age of the population was above 59 years, and yet the findings support the relationship of aspirin use and duration of use with enhanced survival in patients with CRC. Future prospective studies may provide further evidence of the relationship of aspirin as a primary prevention of CRC and its potential benefits for patients already diagnosed with CRC.

\section{Acknowledgements}

This study was a result of several individuals' contributions. Contribution to data compilation was done by Drs. Walter Woznick, Dr. Jonathan Bakos, and Dr. Matthew Brown. Dr. Jasim Albandar is recognized for his invaluable input with editing the final manuscript.

\section{Footnote}

Conflicts of Interest: The authors have no conflicts of interest to declare.

Ethical Statement: The study was approved by the Wright State University Institutional Review Board and the DVAMC Research and Development Committee (No. IRB00000034) and the IRB approved a waiver of informed consent for this retrospective chart review.

\section{References}

1. Ferlay J, Soerjomataram I, Dikshit R, et al. Cancer incidence and mortality worldwide: sources, methods and major patterns in GLOBOCAN 2012. Int J Cancer 2015;136:E359-86.

2. Rahib L, Smith BD, Aizenberg R, et al. Projecting cancer incidence and deaths to 2030: the unexpected burden of thyroid, liver, and pancreas cancers in the United States. Cancer Res 2014;74:2913-21.

3. Espey DK, Wu XC, Swan J, et al. Annual report to the nation on the status of cancer, 1975-2004, featuring cancer in American Indians and Alaska Natives. Cancer 2007;110:2119-52.

4. Edwards BK, Brown ML, Wingo PA, et al. Annual report to the nation on the status of cancer, 1975-2002, featuring 
population-based trends in cancer treatment. J Natl Cancer Inst 2005;97:1407-27.

5. Huxley RR, Ansary-Moghaddam A, Clifton P, et al. The impact of dietary and lifestyle risk factors on risk of colorectal cancer: a quantitative overview of the epidemiological evidence. Int J Cancer 2009;125:171-80.

6. Tantamango-Bartley Y, Jaceldo-Siegl K, Fan J, et al. Vegetarian diets and the incidence of cancer in a lowrisk population. Cancer Epidemiol Biomarkers Prev 2013;22:286-94.

7. Aune D, Chan DS, Lau R, et al. Dietary fibre, whole grains, and risk of colorectal cancer: systematic review and dose-response meta-analysis of prospective studies. BMJ 2011;343:d6617.

8. Rothwell PM, Fowkes FG, Belch JF, et al. Effect of daily aspirin on long-term risk of death due to cancer: analysis of individual patient data from randomised trials. Lancet 2011;377:31-41.

9. Baron JA, Cole BF, Sandler RS, et al. A randomized trial of aspirin to prevent colorectal adenomas. N Engl J Med 2003;348:891-9.

10. Flossmann E, Rothwell PM; British Doctors Aspirin Trial and the UK-TIA Aspirin Trial. Effect of aspirin on long-term risk of colorectal cancer: consistent evidence from randomised and observational studies. Lancet 2007;369:1603-13.

11. Dubé C, Rostom A, Lewin G, et al. The use of aspirin for primary prevention of colorectal cancer: a systematic review prepared for the U.S. Preventive Services Task Force. Ann Intern Med 2007;146:365-75.

12. Fuchs C, Meyerhardt JA, Heseltine DL, et al. Influence of regular aspirin use on survival for patients with stage

Cite this article as: Albandar HJ, Markert R, Agrawal S. The relationship between aspirin use and mortality in colorectal cancer. J Gastrointest Oncol 2018;9(6):1133-1137. doi: 10.21037/jgo.2018.08.13
III colon cancer: findings from intergroup trial CALGB 89803. J Clin Oncol 2005;23:3530.

13. Chan AT, Ogino S, Fuchs CS. Aspirin use and survival after diagnosis of colorectal cancer. JAMA 2009;302:649-58.

14. Rothwell PM, Wilson M, Price JF, et al. Effect of daily aspirin on risk of cancer metastasis: a study of incident cancers during randomised controlled trials. Lancet 2012;379:1591-601.

15. Reimers MS, Bastiaannet E, Langley RE, et al. Expression of HLA class I antigen, aspirin use, and survival after a diagnosis of colon cancer. JAMA Intern Med 2014;174:732-9.

16. Rothwell PM, Price JF, Fowkes FG, et al. Short-term effects of daily aspirin on cancer incidence, mortality, and non-vascular death: analysis of the time course of risks and benefits in 51 randomised controlled trials. Lancet 2012;379:1602-12.

17. McCowan C, Munro AJ, Donnan PT, et al. Use of aspirin post-diagnosis in a cohort of patients with colorectal cancer and its association with all-cause and colorectal cancer specific mortality. Eur J Cancer 2013;49:1049-57.

18. Cardwell CR, Kunzmann AT, Cantwell MM, et al. Lowdose aspirin use after diagnosis of colorectal cancer does not increase survival: A case-control analysis of a populationbased cohort. Gastroenterology 2014;146:700-8.

19. Final Recommendation Statement: Aspirin Use to Prevent Cardiovascular Disease and Colorectal Cancer: Preventive Medication. U.S. Preventive Services Task Force. September 2017. Available online: https://www. uspreventiveservicestaskforce.org/Page/Document/ RecommendationStatementFinal/aspirin-to-preventcardiovascular-disease-and-cancer 